\title{
Electron Diffraction Evidence For An Elastic XY Model Phase In Niobia-Zirconia Ceramic Alloys
}

\author{
J. R. Sellar
}

School of Physics and Materials Engineering, Monash University, 3800 Victoria Australia

It has become increasingly common to describe functional materials as analogues of well-known spin models, describing their properties in terms of a "magnetic language" even when their functional behaviour is not magnetic. Dielectric materials, for example, have been described in terms of the Ising model, high-temperature superconductors in terms of XY models and ionic conductors in terms of lattice gases, which can be mapped directly onto the Ising model. Though possibly an over-simplification in some cases, such model identifications become clearer near phase transitions since the universality class of the transition depends only on the dimensionality of the order parameter and of the space in which it operates.

In the present note, several separate pieces of evidence in the form of electron diffraction patterns and high-resolution electron micrographs will be presented which suggest strongly that the material under investigation (a niobia-"stabilized" zirconia ceramic alloy) is usefully characterised thermodynamically in terms of a frustrated elastic $2 \mathrm{D}$ XY model.

The zirconia-rich niobia-zirconia alloys have been a structural puzzle for a long time. From a diffraction viewpoint, the chief consequence of "stabilizing" zirconia $\left(\mathrm{ZrO}_{2}\right)$ with niobia $\left(\mathrm{Nb}_{2} \mathrm{O}_{5}\right)$ appeared initially to be the production of orthorhombic long-period superlattices with very sharp diffraction spots and an apparently continuous distribution of dopant-dependent superlattice wave vectors. The alloys possessed the formula $\mathrm{Nb}_{2} \mathrm{Zr}_{\mathrm{x}-2} \mathrm{O}_{2 \mathrm{x}+1}(\mathrm{x} \approx 7-10)$, such that for each value of $\mathrm{x}$ a single superlattice phase resulted. Later the alloys were described in terms of two-dimensional sublattice nets of oxygen ions transforming smoothly between locally hexagonal and tetragonal segments, by means of small local rotations of the nets[1]. It is these two-dimensional local rotations of the oxygen anion nets in the $\mathrm{x}-\mathrm{z}$ plane of the alloys which constitute the basis of the claim that the continuous series of niobia-zirconia compounds in the orthorhombic phase are best described by means of an XY model[2].

Using four-dimensional space-group techniques[3], however, the alloys have recently been characterized in the general non-stoichiometric case as incommensurately-modulated structures[4], with separate disordered oxygen and metal substructures[5] and a continuously variable composition-dependent primary modulated wave-vector with sometimes very long incommensurate modulations of the oxygen anions. A crystallographic description of the alloys in terms of fourdimensional space groups is valid only if the oxygen satellite reflections are parallel to the cationic subcell reflections in reciprocal space. In essentially all diffraction patterns from the alloys however, a non-collinear canting of the satellite reflections is found[4], including large angular excursions from the subcell $a^{*}$ reciprocal space direction under conditions of non-equilibrium cooling, which limits such a simple crystalline interpretation of the alloys' diffraction data.

The experimental results to be discussed are the following: 
- the ubiquitous canting of the satellite reflections due to small local rotations of the oxygen ion nets. As previously noted, these two-dimensional rotations are the elastic analogues of XY magnetic spins. The canting is usually small as evidenced in the electron diffraction patterns, but is found in all circumstances.

- Widespread displacive disorder of the oxygen ion nets. Room temperature synchrotron experiments on niobia-zirconia samples result in large values for displacement[5] of the oxygen ions from their average positions, symptomatic of the inability of a 2D XY model to order [6].

- When scaled against the metal sublattice reflections, the oxygen net sublattice reflections appear to give spots of very different sizes, depending on the type of radiation used. Synchrotron X-ray spots were reported to appear "broad and ill-defined" in reciprocal space[7] whereas the corresponding electron diffraction satellite spots are usually extremely sharp, as already mentioned. This behaviour is consistent with the alloys being cooled below the KosterlitzThouless transformation temperature where the XY model is considered to be in a critical state exhibiting quasi-long-range order (QLRO) with a power-law decay of order-parameter correlation rather than a single well-defined half-width[8]. In the critical phase, the apparent reciprocal space profile is that of sharp spots without any definite width, which depends instead on the brightness and coherence of the probe radiation and the relative strength of he scatter.

- Extra spots appear in the electron diffraction patterns from the alloys. This effect is believed to be due to correlations frozen-in during quenching: at criticality density fluctuations of essentially all wavelengths coexist in an XY phase, so essentially any frozen-in modulation is possible.

- In the XY Kosterlitz-Thouless transition the phase above the transition temperature is considered to be a two-dimensional liquid[8]. The transition is mediated by dislocation-like vortices which have been detected in several electron micrographs taken from quenched alloy samples.

- On its own, the XY model cannot explain the phenomenon of satellite spot canting. Electron micrographs and diffraction patterns will be presented displaying the quasi-periodic coherent intergrowth of oppositely-canted chiral domains in alloy grains. This is believed to be an analogue of the effect of frustration on a 2D magnetic XY model on a square lattice.

\section{References}

[1] B.G. Hyde, A.N. Bagshaw, S. Andersson and M.O’Keeffe, Ann. Rev. Mat. Sci. 4 (1974) 43

[2] J.R. Sellar, Micron 32 (2001) 871

[3] T. Janssen, A. Janner, A. Looijenga-Vos and P.M. de Wolff, International Tables for Crystallography Vol.C (ed.A.J.C. Wilson), (1992) 797

[4] J.G. Thompson, R.L. Withers, J.R. Sellar, P.J. Barlow and B.G. Hyde, J. Solid State Chem. 88 (1990) 465

[5] S. Schmid, J.G. Thompson, R.L. Withers, V. Petricek, N. Ishizawa, S. Kishimoto, Acta Cryst. B53 (1997) 851

[6] N. D. Mermin and H. Wagner, Phys. Rev. Letts. 17 (1966) 1133

[7] K. Futterer, S. Schmid, J.G. Thompson, R.L. Withers, N. Ishizawa and S. Kishimoto, Acta Cryst. B51 (1995) 680

[8] J.M. Kosterlitz and D.J. Thouless, J. Phys. C 6 (1973) 1181 